\title{
Adherence to the Mediterranean diet is associated with the gut microbiota pattern and gastrointestinal characteristics in an adult population
}

\author{
Evdokia K. Mitsou ${ }^{1}$, Aimilia Kakali ${ }^{1}$, Smaragdi Antonopoulou ${ }^{1}$, Konstantinos C. Mountzouris ${ }^{2}$, \\ Mary Yannakoulia ${ }^{1}$, Demosthenes B. Panagiotakos ${ }^{1}$ and Adamantini Kyriacou ${ }^{1 *}$ \\ ${ }^{1}$ Department of Nutrition and Dietetics, Harokopio University, 70 El. Venizelou str., 17671 Kallithea, Greece \\ ${ }^{2}$ Department of Nutritional Physiology and Feeding, Agricultural University of Athens, 75 Iera Odos, 11855 Athens, Greece
}

(Submitted 14 November 2016 - Final revision received 27 March 2017 - Accepted 5 June 2017)

\section{Abstract}

This study aimed to explore the potential associations of adherence to the Mediterranean diet with gut microbiota characteristics and gastrointestinal symptomatology in an adult population. Other long-term dietary habits (e.g. consumption of snacks and junk food or stimulant intake) were also evaluated in terms of the gut microbiota profile. Participants ( $n$ 120) underwent anthropometric, dietary, physical activity and lifestyle evaluation. Adherence to the Mediterranean diet was assessed using a Mediterranean diet score, the MedDietScore, and subjects were classified into three tertiles according to individual adherence scoring. Gut microbiota composition was determined using quantitative PCR and plate-count techniques, and faecal SCFA were analysed using GC. Gastrointestinal symptoms were also evaluated. Participants with a high adherence to the Mediterranean diet had lower Escherichia coli counts $(P=0 \cdot 022)$, a higher bifidobacteria:E. coli ratio $(P=0 \cdot 025)$, increased levels and prevalence of Candida albicans $(P=0.039$ and $P=0.050$, respectively), greater molar ratio of acetate $(P=0.009)$, higher defaecation frequency $(P=0.028)$ and a more pronounced gastrointestinal symptomatology compared with those reporting low adherence. A lower molar ratio of valerate was also observed in the case of high adherence to the Mediterranean diet compared with the other two tertiles ( $P_{\text {for trend }}=0 \cdot 005$ ). Positive correlations of MedDietScore with gastrointestinal symptoms, faecal moisture, total bacteria, bifidobacteria:E. coli ratio, relative share of Bacteroides, $C$. albicans and total SCFA, as well as negative associations with cultivable $E$. coli levels and valerate were indicated. Fast food consumption was characterised by suppressed representation of lactobacilli and butyrate-producing bacteria. In conclusion, our findings support a link between adherence to the Mediterranean diet and gut microbiota characteristics.

Key words: Gut microbiota: Mediterranean diet: Junk foods: Yeasts: SCFA

The gut microbiota is a diverse and dynamic microbial ecosystem, which supports important gastrointestinal and systemic metabolic functions of the host ${ }^{(1,2)}$. Nowadays, the gut microbiota is increasingly being accepted as a novel environmental factor associated with the occurrence and progress of various pathological conditions, including obesity and related metabolic comorbidities as well as cancer and inflammatory and degenerative diseases ${ }^{(1-3)}$. Though several gut micro-organisms (e.g. Faecalibacterium prausnitzii, Akkermansia muciniphila, Methanobrevibacter smithii) have emerged as microbial indicators of metabolic health and inflammation ${ }^{(4-6)}$, more research is necessary to elucidate the potential role of intestinal microbiota in health and disease, taking into consideration the potential effect of lifestyle patterns, including diet ${ }^{(2)}$. Long-term dietary habits have a considerable effect on the human gut microbiota, and epidemiological data have already indicated connections between diet constituents or dietary patterns and gut microbiota profile and functionality ${ }^{(7)}$.
The Mediterranean diet is a healthy dietary pattern, inspired by the food patterns of populations living around the Mediterranean Sea in the early $1960 \mathrm{~s}^{(8,9)}$. Its characteristic features are high consumption of fruits, vegetables, legumes, unrefined cereals and nuts, moderate consumption of fish, poultry and dairy products (principally cheese and yogurt), low consumption of red meat products, use of olive oil as the main edible-fat source and regular but moderate wine consumption ${ }^{(9)}$. Greater adherence to the Mediterranean diet has been linked to a significant reduction in overall mortality and morbidity, inspiring a beneficial dietary approach in the management of CVD, type 2 diabetes, obesity, inflammatory diseases, degenerative diseases and cancer ${ }^{(3,8,10)}$. In addition to the proposed protective mechanisms of the Mediterranean diet against major chronic diseases (e.g. antiinflammatory, antioxidant, satiogenic and fat-oxidation effects) ${ }^{(11)}$, this dietary pattern has recently generated interest regarding the manipulation of gut microbiota characteristics in the battle against inflammatory and metabolic disorders ${ }^{(3,10-19)}$.

Abbreviation: qPCR, quantitative PCR.

* Corresponding author: A. Kyriacou, fax +30 210 9577050, email mkyriacou@hua.gr 
The aim of this cross-sectional study was to elucidate the potential associations of adherence to the Mediterranean diet with gut microbiota characteristics and gastrointestinal symptomatology in an adult population. We particularly focused on proposed microbial indicators of inflammation and metabolic health and on suggested diet-responsive groups of bacteria (e.g. Prevotella, Roseburia-Eubacterium rectale, Clostridium coccoides group) ${ }^{(4-7)}$. Further, we have determined additional members of faecal microbiota, such as Candida spp., with rather unexplored contributions. Other long-term dietary habits (e.g. consumption of snacks and junk food or stimulant intake) were also evaluated in terms of the gut microbiota profile.

\section{Methods}

\section{Study population}

A total of 120 participants were recruited from Athens, Greece, during the period 2011-2015. Volunteer recruitment was carried out through word of mouth and local press announcements. Eligible participants were men and women, aged 18-65 years, without a history of gastrointestinal disease, autoimmune disease, coronary disease, liver and/or kidney malfunction, epileptic seizures, without current inflammation, present pregnancy, recent weight loss, extreme dietary behaviours, no consumption of antibiotics 2 months before the study and/or intake of non-steroid anti-inflammatory agents, antioxidant and $n-3$ supplements, probiotic and/or prebiotic supplements 2 weeks before the study.

\section{Ethical standards}

This study was conducted according to the guidelines laid down in the Declaration of Helsinki and all procedures involving human subjects were approved by the Bioethics Committee of Harokopio University. Written informed consent was obtained from all subjects before their inclusion in the study.

\section{Demographic and clinical information}

Subjects completed a series of questionnaires in relation to sociodemographic parameters (including age, sex, marital status and education level), smoking habits, medical history, psychological parameters ${ }^{(20)}$ and sleeping patterns. Blood pressure $(\mathrm{mmHg})$ and heart rate of fasted volunteers were measured in the sitting position.

\section{Anthropometry}

Body weight and height were measured on a levelled platform scale (SECA GmbH) and a wall-mounted stadiometer (SECA $\mathrm{GmbH}$ ) to the nearest $0.1 \mathrm{~kg}$ and $0.5 \mathrm{~cm}$, respectively. BMI was calculated by dividing the weight $(\mathrm{kg})$ by the height $\left(\mathrm{m}^{2}\right)$. Waist circumference was measured in the middle between the twelfth rib and the iliac crest, and hip circumference was measured at the widest lateral extension of the hips to the nearest $0.1 \mathrm{~cm}$ using a measuring tape. Waist:hip ratio was also calculated. Triceps skinfold was measured at the midpoint between the olecranon process and the acromion process using a Lange
Skinfold Caliper (Beta Technology Incorporated). Anthropometric evaluation also included body analysis assessment using bioelectrical impedance (BC-418 Segmental Body Composition Analyzer; Tanita).

\section{Dietary assessment}

Dietary intake was evaluated using a validated semi-quantitative $F F Q^{(21)}$. The FFQ was completed by each participant with the aid of an experienced investigator. Collected data were then analysed in terms of energy and nutrient intakes using the Nutritionist Pro software (version 4.1.0; Axxya Systems). In all, thirteen main food groups (dairy products, starchy products, eggs, meat, fish, legumes, vegetables, fruit, fats and oils, sweets, alcoholic beverages, snacks and junk food, stimulants) were also created, and servings per day $/ 4184 \mathrm{~kJ}$ (1000 kcal) of energy intake (EI) for each food group was calculated. The level of adherence to the Mediterranean diet was assessed using an eleven-item composite index, the MedDietScore ${ }^{(22)}$. For food items presumed to be close to the Mediterranean dietary pattern (i.e. those that are suggested to be consumed on a daily basis or in >4 servings/week: non-refined cereals, fruit, vegetables, potatoes, legumes, olive oil and fish), a score of 0 was assigned when a participant reported no consumption, a score of 1 for reported consumption of 1-4 times/month, a score of 2 for 5-8 times/month, a score of 3 for 9-12 times/month, a score of 4 for 13-18 times/month and a score of 5 for $>18$ times/month. On the contrary, for the consumption of foods presumed to be away from this dietary pattern (i.e. those suggested to not be consumed on a daily or weekly basis: meat and meat products, poultry and high-fat dairy products), the opposite scores were assigned (i.e. a score of 0 when a participant reported almost daily consumption of the food to a score of 5 for rare or no consumption). For alcohol consumption, a non-monotonic scoring was adopted based on daily intake of $15-30 \mathrm{~g}$ ethanol as suggested by the Mediterranean dietary pattern (i.e. a score of 5 was assigned for consumption of $<3$ glasses/d; 0 for none or consumption of $>7$ glasses/d; and scores of $4,3,2$ and 1 for the consumption of 3, 4-5, 6 and 7 glasses/d, respectively). A total score was calculated on the basis of these eleven components (score range: 0-55); higher score values indicate a greater adherence to the Mediterranean diet, whereas lower score values indicate adherence to a Westernised diet. On the basis of calculated values of MedDietScore in our study, the tertiles of this score were applied for classification of individuals into three different groups for further analysis ${ }^{(22,23)}$.

For the assessment of low energy reporting, the ratio of the EI:BMR was determined for each subject. BMR was estimated using the Schofield equations as adopted by the $\mathrm{WHO}^{(24)}$. Participants with EI:BMR $\leq 1 \cdot 13$ were classified as 'low energy reporters' (LER), on the basis of the methodology developed by Goldberg et al. ${ }^{(25)}$.

\section{Physical activity assessment}

Physical activity levels were assessed using the International Physical Activity Questionnaire Short Form questionnaire validated for the Greek population ${ }^{(26)}$. Duration of sedentary activity (sitting or resting) expressed as 'h/week' was also recorded. 


\section{Evaluation of gastrointestinal symptoms}

The intensity of gastrointestinal symptoms (i.e. abdominal pain, bloating, flatulence, borborygmi) was recorded on a weekly basis and measured daily on a scale of $0-4$, where ' 0 ' represented absence of symptoms and ' 4 ' severe symptoms. Gastrointestinal symptoms were evaluated as a 7-d symptom score and the total weekly symptom score was calculated as the sum of the four symptom scores ${ }^{(27)}$. Stool frequency and consistency of evacuations using the Bristol Stool Scale were also recorded ${ }^{(28)}$. This stool-form scale could also serve as a useful guide to intestinal transit time ${ }^{(28)}$. Participants were further asked about gastrointestinal pain and the mean number of daily evacuations for the 4-week period preceding the study ${ }^{(29)}$

\section{Stool collection}

Participants were given a faecal collection kit with a sterile stool tube (Oxoid AnaeroGen ${ }^{\mathrm{TM}}$; Thermo Scientific Inc.) and a preweighed plastic container to return their faecal sample in during the next few days. Stool samples were processed under anaerobic conditions for plate-count techniques within $2 \mathrm{~h}$ after defaecation, or homogenised and stored immediately at $-80^{\circ} \mathrm{C}$ for future molecular analysis.

\section{Gut microbiota analysis}

Enumeration of gut microbiota was performed using both platecount techniques (online Supplementary Table S1) and real-time quantitative PCR (qPCR). Colony counts were expressed as a $\log _{10}$ of the colony-forming units/g wet faeces. The relative share (\%) of each microbial group was calculated ${ }^{(30)}$ and detection frequencies were also estimated. For molecular analysis, genomic DNA was extracted according to Salonen et al.$^{(31)}$ using QIAamp ${ }^{\circledR}$ DNA Mini Kit (QIAGEN GmbH). Quantitative real-time PCR based on SYBR Green I detection chemistry was used to characterise the gut microbiota using species-, genus- and group-specific primers targeting 16S rRNA genes of different bacterial groups or the nuc gene in the case of Staphylococcus aureus (online Supplementary Table S2) and the KAPA SYBR ${ }^{\circledR}$ Fast Master Mix (2X) Universal Kit (Kapa Biosystems Inc.). PCR amplification and detection were performed in a LightCycler ${ }^{\circledR} 2.0$ Real-Time PCR System (Roche Diagnostics $\mathrm{GmbH}$ ). Microbial quantification was based on standard curves of genomic DNA from reference strains with the LightCycler® software version 4.1 (Roche Diagnostics $\mathrm{GmbH}$ ). Data are expressed as $\log _{10}$ copies of $16 \mathrm{~S}$ rRNA gene/g wet faeces or $\log _{10}$ copies of nuc gene/g wet faeces in the case of $S$. aureus.

\section{Faecal SCFA and pH determination}

Faecal SCFA concentrations were determined using capillary GC, as previously described ${ }^{(32)}$, after 1:3 dilution of frozen faecal samples $\left(1.5 \mathrm{~g},-80^{\circ} \mathrm{C}\right)$ using $0.9 \%$ saline. Faecal $\mathrm{pH}$ of fresh samples and stool moisture were also determined ${ }^{(33)}$.

\section{Statistical analysis}

Normality of the distribution of variables was tested using the Kolmogorov-Smirnov test. Comparisons of normally distributed variables between study groups were performed using one-way
ANOVA and univariate ANCOVA, whereas for skewed variables the Kruskal-Wallis $H$ test and rank ANCOVA were used, as appropriate; Bonferroni's correction rule for the inflation of type I error was applied. The $\chi^{2}$ was applied for checking dependency between categorical variables; whereas Pearson's $r$ or Spearman's $\rho$ correlation coefficients were used to evaluate linear relationships among continuous variables. Multiple linear regression analysis (presented as $\beta$-coefficients with their standard errors and $P$ values) and logistic regression analysis were performed to evaluate MedDietScore or specific food groups (independent variable) in relation to faecal, gut microbiota and gastrointestinal characteristics (dependent outcomes) of the participants. The statistical analysis was performed using SPSS $^{\circledR}$ Statistical software version 21 (IBM Hellas). The sample size of the present study was defined $a$ priori in order to evaluate 0.5 standardised differences between Mediterranean diet adherers and non-adherers in various bacteria and SCFA studied in the present protocol. In particular, to achieve $80 \%$ statistical power at a 5\% significance level of two-sided hypotheses, fifty-eight participants were considered adequate to evaluate the aforementioned differences.

\section{Results}

A total of 116 subjects (sixty-one male and fifty-five female; mean age 42 years) completed the study. Dropout $(n=4)$ was because of failure of faecal sampling. LER represented $13.8 \%$ of the sample (sixteen subjects; nine obese, six overweight, one normal weight) and they were excluded from the analyses below. On the basis of values of MedDietScore, subjects in our study ( $n$ 100) were classified into three tertiles of adherence to the Mediterranean diet according to individual MedDietScore (low tertile (score 19.0-30·0, $n$ 31), medium tertile (score 31.0-33.0, $n$ 29) and high tertile (score 34.0-41.0, $n$ 40)). Descriptive characteristics of the study participants according the tertile of adherence to the Mediterranean diet are available in Table 1.

\section{Diet, physical activity and sleeping patterns}

Participants with the highest adherence to the Mediterranean diet reported higher consumption of starchy products, vegetables, fruits, fish and eggs, but lower consumption of meat, snacks and stimulants (e.g. coffee/tea, sodas) compared with those in the low tertile (online Supplementary Table S3). No significant difference was detected among MedDietScore categories in levels of physical activity, total physical activity score, sedentary lifestyle and sleeping duration with or without adjustment for sex, age and BMI (online Supplementary Table S4).

\section{Gastrointestinal symptoms and evacuation characteristics}

Subjects in the high-adherence tertile reported greater total number of evacuations during the $7-\mathrm{d}$ period compared with the low-adherence tertile $(P=0.028)$ (Table 2$)$. Though no significant differences were detected among tertiles in terms of Bristol stool scale values, there was a trend for lower scale rating in the participants of medium tertile compared with those 
Table 1. Subjects' basic characteristics

(Mean values and standard deviations; medians and quartiles 1-3 (Q1-Q3))

\begin{tabular}{|c|c|c|c|c|c|c|c|c|c|}
\hline & & & \multicolumn{6}{|c|}{ Tertiles of MedDietScore } & \multirow[b]{3}{*}{$P_{\text {for trend }}$} \\
\hline & \multicolumn{2}{|c|}{ Total $(n 100)$} & \multicolumn{2}{|c|}{ Low $(n 31)$} & \multicolumn{2}{|c|}{ Medium ( $n$ 29) } & \multicolumn{2}{|c|}{ High $(n 40)$} & \\
\hline & Mean & SD & Mean & SD & Mean & SD & Mean & SD & \\
\hline \multicolumn{10}{|l|}{ Sociodemographic parameters } \\
\hline Age (years) & 41.27 & 13.33 & $40 \cdot 42$ & $13 \cdot 12$ & 40.14 & $13 \cdot 16$ & $42 \cdot 75$ & $13 \cdot 33$ & 0.657 \\
\hline Sex (male/female) & $48 / 52$ & & $19 / 12^{a}$ & & $15 / 14^{a, b}$ & & $14 / 26^{b}$ & & 0.080 \\
\hline Years of education & 17.05 & 4.77 & $17 \cdot 18$ & 5.00 & $17 \cdot 16$ & 5.02 & $16 \cdot 87$ & 4.52 & 0.953 \\
\hline Marital status (no. of singles) & \multicolumn{2}{|c|}{51} & \multicolumn{2}{|c|}{16} & \multicolumn{2}{|c|}{14} & \multicolumn{2}{|c|}{15} & 0.453 \\
\hline Current smoking (no. of smokers) & \multicolumn{2}{|c|}{31} & \multicolumn{2}{|c|}{14} & \multicolumn{2}{|c|}{7} & \multicolumn{2}{|c|}{10} & 0.121 \\
\hline \multicolumn{10}{|l|}{ Medical and psychological status } \\
\hline $\mathrm{SBP}(\mathrm{mmHg})$ & $118 \cdot 86$ & $16 \cdot 24$ & $118 \cdot 15$ & $18 \cdot 25$ & 121.97 & $10 \cdot 67$ & $117 \cdot 15$ & 17.93 & 0.462 \\
\hline $\mathrm{DBP}(\mathrm{mmHg})$ & $76 \cdot 32$ & $10 \cdot 44$ & 76.60 & $10 \cdot 38$ & 76.90 & 8.28 & 75.67 & 11.98 & 0.878 \\
\hline Heart rate (beats/min) & 73.66 & $10 \cdot 04$ & 76.48 & $9 \cdot 12$ & 72.43 & $10 \cdot 72$ & $72 \cdot 32$ & 10.01 & 0.167 \\
\hline Self-evaluated health status score (score $0.0-100.0$ ) & & & & & & & & & 0.846 \\
\hline Median & \multicolumn{2}{|c|}{$85 \cdot 0$} & \multicolumn{2}{|c|}{80.0} & \multicolumn{2}{|c|}{$85 \cdot 0$} & \multicolumn{2}{|c|}{$85 \cdot 0$} & \\
\hline Q1-Q3 & \multicolumn{2}{|c|}{$80 \cdot 0-90 \cdot 0$} & \multicolumn{2}{|c|}{$78 \cdot 8-90 \cdot 0$} & \multicolumn{2}{|c|}{$75 \cdot 0-90 \cdot 0$} & \multicolumn{2}{|c|}{$80 \cdot 0-90 \cdot 0$} & \\
\hline ZDRS score (score $20 \cdot 0-80 \cdot 0$ ) & 33.41 & $6 \cdot 87$ & 33.90 & $7 \cdot 10$ & 33.39 & $6 \cdot 21$ & 33.05 & $7 \cdot 26$ & 0.879 \\
\hline STAI score (score $20 \cdot 0-80 \cdot 0$ ) & 34.76 & 9.07 & $35 \cdot 17$ & 9.57 & $36 \cdot 11$ & 9.02 & 33.50 & 8.78 & 0.489 \\
\hline \multicolumn{10}{|l|}{ Anthropometric measurements } \\
\hline Weight $(\mathrm{kg})$ & $77 \cdot 48$ & $15 \cdot 31$ & 78.44 & 17.05 & 80.48 & $12 \cdot 38$ & 74.56 & $15 \cdot 64$ & 0.262 \\
\hline Height $(m)$ & 1.68 & 0.09 & 1.69 & 0.10 & 1.70 & 0.08 & 1.66 & 0.09 & 0.134 \\
\hline $\operatorname{BMI}\left(\mathrm{kg} / \mathrm{m}^{2}\right)$ & $27 \cdot 29$ & 4.48 & $27 \cdot 46$ & 4.80 & 27.68 & 3.66 & $26 \cdot 88$ & 4.83 & 0.743 \\
\hline WC (cm) & $90 \cdot 08$ & 13.46 & $91 \cdot 34$ & 14.01 & 92.48 & $10 \cdot 58$ & 87.49 & 14.59 & 0.273 \\
\hline $\mathrm{HC}(\mathrm{cm})$ & 107.99 & 8.20 & $108 \cdot 10$ & 8.40 & 109.93 & $6 \cdot 31$ & $106 \cdot 61$ & 9.06 & 0.270 \\
\hline WHR & 0.83 & 0.09 & 0.84 & 0.09 & 0.84 & 0.08 & 0.82 & 0.10 & 0.425 \\
\hline Triceps skinfold (mm) & 24.02 & $6 \cdot 46$ & 23.59 & $6 \cdot 61$ & 24.69 & 7.34 & 23.90 & $5 \cdot 80$ & 0.805 \\
\hline Body fat (\%) & $29 \cdot 11$ & 8.78 & $27 \cdot 42$ & 8.92 & \multirow{2}{*}{\multicolumn{2}{|c|}{29.46}} & $30 \cdot 16$ & 8.06 & 0.418 \\
\hline FFM $(\mathrm{kg})$ & \multirow{2}{*}{\multicolumn{2}{|c|}{51.70}} & \multirow{2}{*}{\multicolumn{2}{|c|}{$59 \cdot 90^{\mathrm{a}, \mathrm{b}}$}} & & & & & \multirow[t]{3}{*}{0.081} \\
\hline Median & & & & & 57 & & & & \\
\hline Q1-Q3 & 43.80 & 34.20 & 43.60 & 6.50 & $45 \cdot 80$ & $7 \cdot 60$ & 43.00 & 1.40 & \\
\hline
\end{tabular}

SBP, systolic blood pressure; DBP, diastolic blood pressure; ZDRS, Zung Depression Rating Scale; STAI, State-Trait Anxiety Inventory; WC, waist circumference; HC, hip circumference; WHR, waist:hip ratio; FFM, fat-free mass.

a,b Mean or median values within a row with unlike superscript letters were significantly different $(P<0.05)$.

Table 2. Gastrointestinal symptoms and evacuation characteristics

(Medians and quartiles 1-3 (Q1-Q3))

\begin{tabular}{|c|c|c|c|c|c|c|c|}
\hline & \multicolumn{6}{|c|}{ Tertiles of MedDietScore } & \multirow[b]{3}{*}{$P_{\text {for trend }}$} \\
\hline & \multicolumn{2}{|c|}{ Low $(n 31)$} & \multicolumn{2}{|c|}{ Medium (n 29) } & \multicolumn{2}{|c|}{ High $(n 40)$} & \\
\hline & Median & Q1-Q3 & Median & Q1-Q3 & Median & Q1-Q3 & \\
\hline \multicolumn{8}{|l|}{ During the last 4 weeks } \\
\hline Evacuations (no./d)* & & & & & & & 0.388 \\
\hline$<1$ & \multicolumn{2}{|r|}{6} & \multicolumn{2}{|c|}{5} & \multicolumn{2}{|c|}{9} & \\
\hline 1 & \multicolumn{2}{|c|}{19} & \multicolumn{2}{|c|}{22} & \multicolumn{2}{|c|}{19} & \\
\hline 2 & \multicolumn{2}{|r|}{4} & \multicolumn{2}{|c|}{1} & \multicolumn{2}{|c|}{9} & \\
\hline 3 & \multicolumn{2}{|r|}{1} & \multicolumn{2}{|c|}{1} & \multicolumn{2}{|c|}{2} & \\
\hline$\geq 4$ & \multicolumn{2}{|r|}{0} & \multicolumn{2}{|c|}{0} & \multicolumn{2}{|c|}{1} & \\
\hline Abdominal or epigastric pain (score $0.00-10.00)^{*}$ & 0.00 & $0.00-0.63$ & 0.00 & $0.00-0.76$ & 0.00 & $0.00-1.09$ & $0.930 \dagger$ \\
\hline \multicolumn{8}{|l|}{ 7-d symptom questionnaire $\ddagger$} \\
\hline Evacuations (no./week) & $7 \cdot 00^{\mathrm{a}}$ & $5 \cdot 75-9 \cdot 00$ & $7 \cdot 50^{a, b}$ & $6 \cdot 25-9 \cdot 00$ & $9 \cdot 00^{\mathrm{b}}$ & $6 \cdot 00-11 \cdot 00$ & $0.078 \dagger$ \\
\hline Diarrhoeic evacuations (no./week) & 0.00 & $0.00-0.00$ & 0.00 & $0.00-0.00$ & 0.00 & $0.00-1.00$ & $0.678 \dagger$ \\
\hline Bristol scale (range $1.0-7.0$ ) & $4 \cdot 0$ & $3 \cdot 0-4 \cdot 0$ & 3.0 & $3.0-4.0$ & $4 \cdot 0$ & $3 \cdot 0-4 \cdot 0$ & $0.224 \dagger$ \\
\hline Faecal colour (range 1.0-4.0) & 3.00 & $2.75-3.00$ & 3.00 & $2 \cdot 00-3 \cdot 00$ & 3.00 & $2.00-3.00$ & $0.497 \dagger$ \\
\hline Faecal smell (range 1.0-4.0) & $2 \cdot 00$ & $1.00-2.00$ & 2.00 & $1 \cdot 25-2 \cdot 75$ & $2 \cdot 00$ & $1.00-3.00$ & $0.305 \dagger$ \\
\hline Abdominal pain (score 0.0-28.0) & $0.00^{\mathrm{a}}$ & $0.00-2.00$ & $0.50^{a, b}$ & $0.00-2 \cdot 75$ & $2 \cdot 00^{\mathrm{b}}$ & $0.00-4.00$ & $0.087 \dagger$ \\
\hline Bloating (score 0.0-28.0) & $0.00^{\mathrm{a}}$ & $0.00-3.25$ & $0.50^{a, b}$ & $0.00-5.00$ & $4 \cdot 00^{\mathrm{b}}$ & $0.00-6.25$ & $0.066 \dagger$ \\
\hline Flatulence (score 0.0-28.0) & 1.00 & $0.00-7.50$ & 5.00 & $1 \cdot 00-8 \cdot 75$ & $6 \cdot 00$ & $1.00-11 \cdot 25$ & $0.379 \dagger$ \\
\hline Borborygmi (score 0.0-28.0) & 0.00 & $0.00-2.00$ & 0.00 & $0.00-3.75$ & 1.00 & $0.00-5.00$ & $0.302 \dagger$ \\
\hline Sum of symptoms (score $0 \cdot 0-112 \cdot 0$ ) & 8.50 & $1 \cdot 75-15 \cdot 25$ & 11.00 & $6 \cdot 00-16 \cdot 75$ & 14.50 & $6 \cdot 75-26 \cdot 00$ & $0.145 \dagger$ \\
\hline
\end{tabular}

a,b Median values within a row with unlike superscript letters were significantly different $(P<0.05)$.

${ }^{*}$ For evacuations (no./d) and abdominal or epigastric pain: $N 99$ (low tertile: $n$ 30, medium tertile: $n$ 29, high tertile: $n$ 40).

† Sex-, age- and BMl-adjusted $P$ values.

‡ For 7-d symptom questionnaire: $n 96$ (low tertile: $n 30$, medium tertile: $n 28$, high tertile: $n 38$ ). 
in the high tertile $(P=0.085)$, implying a difference in gut transit time between these groups. In terms of gastrointestinal symptoms, scores of pain $(P=0.029)$ and bloating were higher $(P=0.028)$ and the sum of symptoms tended to be higher $(P=0.052)$ in the high compared with the low tertile. The majority of subjects (89\%) experienced mild total gastrointestinal symptomatology, with flatulence being the most commonly reported symptom.

\section{Faecal SCFA, stool pH and moisture content}

Faecal moisture (\%) tended to be higher in the high compared with the low tertile $(P=0.056)$ (Table 3$)$. Total levels of SCFA differed significantly among adherence groups, with detection of lower levels in the case of medium compared with high $(P=0.013)$ and low tertiles $(P=0.078)$, whereas no significant difference was detected between adherence groups in terms of stool pH (Table 3). High adherence to the Mediterranean diet was characterised by a significantly greater molar ratio of acetate compared with the low tertile $(P=0.009)$ and medium tertile $(P=0.075)$, whereas valerate was detected in a lower ratio in the high tertile compared with both medium $(P=0.002)$ and low tertiles $(P=0 \cdot 014)$.

\section{Gut microbiota analysis}

Enumeration of gut microbiota by plate-count techniques and detection frequency of each microbial group are presented in Table 4. Data concerning bacteria, yeasts and fungi are available for ninety-two cases, whereas data for Candida genus and species are available for the entire cohort ( $n$ 100). Relative shares of each microbial group were also calculated on the basis of total cultured bacteria or eukaryotes (data not shown).

Gut microbiota profiling analysis revealed significantly lower counts of Escherichia coli $(P=0.022)$ in the high- compared with the low-adherence group after adjustment for sex, age and BMI (Table 4). This fact further explained the notable difference in bifidobacteria: $E$. coli ratio between these groups $(P=0.025)$, though no significant differentiation could be detected between groups in terms of bifidobacteria levels (Table 4). Counts of Candida albicans were higher in the high tertile compared with the low- $(P=0.039)$ and medium- $(P=0.093)$ MedDietScore groups and a higher detection frequency was observed in the high tertile compared with the low-adherence group (42.5 $\mathrm{v}$. $22.6 \%, P=0.050)$, though no signs or symptoms of candidiasis were reported among subjects (Table 4 ).

No significant differences were observed between tertiles with respect to qPCR analysis after sex, age and BMI adjustment (Table 5).

\section{Associations of the Mediterranean diet with gut microbiota, faecal and gastrointestinal characteristics}

Bivariate models revealed several significant correlations between adherence to the Mediterranean diet, as evaluated by MedDietScore, and gastrointestinal, stool and gut microbiota characteristics, which remained significant even after adjustment for sex, age and

Table 3. Faecal total SCFA concentration, molar ratios of SCFA and stool characteristics

(Mean values and standard deviations; medians and quartiles 1-3 (Q1-Q3))

\begin{tabular}{|c|c|c|c|c|c|c|c|}
\hline & \multicolumn{6}{|c|}{ Tertiles of MedDietScore } & \multirow[b]{3}{*}{$P_{\text {for trend }}{ }^{*}$} \\
\hline & \multicolumn{2}{|c|}{ Low $(n 31)$} & \multicolumn{2}{|c|}{ Medium ( $n$ 29) } & \multicolumn{2}{|c|}{ High ( $n$ 40) } & \\
\hline & Mean & SD & Mean & SD & Mean & SD & \\
\hline Total SCFA ( $\mu \mathrm{mol} / \mathrm{g}$ wet faeces) & $83 \cdot 07^{\mathrm{a}, \mathrm{b}}$ & $35 \cdot 36$ & $67.52^{\mathrm{a}}$ & $25 \cdot 45$ & $83.02^{b}$ & 34.24 & 0.040 \\
\hline \multicolumn{8}{|l|}{ Molar ratios of SCFA } \\
\hline Acetate $(\%)$ & $45 \cdot 41^{\mathrm{a}}$ & 4.92 & $46 \cdot 62^{a, b}$ & $5 \cdot 60$ & $49 \cdot 16^{b}$ & 4.96 & 0.026 \\
\hline Propionate (\%) & $19 \cdot 29$ & $5 \cdot 22$ & 17.69 & 3.64 & $17 \cdot 39$ & 4.91 & 0.245 \\
\hline Butyrate (\%) & $27 \cdot 74$ & 6.09 & $27 \cdot 15$ & 5.82 & 26.77 & 5.43 & 0.928 \\
\hline $\begin{array}{l}\text { Branched-chain SCFA (\%)† } \\
\text { Iso-butyrate (\%) }\end{array}$ & $3 \cdot 70$ & 1.93 & $4 \cdot 28$ & 1.67 & 3.57 & $2 \cdot 34$ & $\begin{array}{l}0.300 \\
0.462\end{array}$ \\
\hline Median & \multirow{2}{*}{\multicolumn{2}{|c|}{$\begin{array}{c}1 \cdot 31 \\
0.76-2 \cdot 24\end{array}$}} & \multirow{2}{*}{\multicolumn{2}{|c|}{$\begin{array}{c}1.38 \\
0.89-2.58\end{array}$}} & \multirow{2}{*}{\multicolumn{2}{|c|}{$\begin{array}{c}1 \cdot 21 \\
0 \cdot 66-2 \cdot 01\end{array}$}} & \\
\hline Q1-Q3 & & & & & & & \\
\hline Iso-valerate (\%) & $2 \cdot 27^{a, b}$ & 1.40 & $2 \cdot 47^{\mathrm{a}}$ & $1 \cdot 22$ & $1.91^{\mathrm{b}}$ & 1.26 & 0.099 \\
\hline Iso-caproic acid (\%) & & & & & & & $0 \cdot 180$ \\
\hline Median & \multicolumn{2}{|c|}{0.00} & \multicolumn{2}{|c|}{0.00} & \multicolumn{2}{|c|}{0.00} & \\
\hline Q1-Q3 & \multicolumn{2}{|c|}{$0.00-0.00$} & \multicolumn{2}{|c|}{$0.00-0.08$} & \multicolumn{2}{|c|}{$0.00-0.07$} & \\
\hline Other SCFA (\%)‡ & $3.49^{\mathrm{a}, \mathrm{b}}$ & 1.69 & $4 \cdot 25^{\mathrm{a}}$ & 1.59 & $3 \cdot 11^{\mathrm{b}}$ & 1.36 & 0.012 \\
\hline Valerate $(\%)$ & $2 \cdot 43^{\mathrm{a}}$ & 0.93 & $2.56^{a}$ & 0.76 & $1.97^{\mathrm{b}}$ & 0.75 & 0.005 \\
\hline Caproic acid (\%) & $0.92^{\mathrm{a}}$ & 0.81 & $1.41^{\mathrm{b}}$ & 0.85 & $0.99^{\mathrm{a}}$ & 0.78 & 0.045 \\
\hline Heptanoic acid (\%) & & & & & & & 0.023 \\
\hline Median & \multicolumn{2}{|c|}{$0.08^{a}$} & \multicolumn{2}{|c|}{$0.20^{\mathrm{b}}$} & \multicolumn{2}{|c|}{$0.13^{a, b}$} & \\
\hline Q1-Q3 & \multicolumn{2}{|c|}{$0.00-0.20$} & \multicolumn{2}{|c|}{$0.11-0.31$} & \multicolumn{2}{|c|}{$0.00-0.24$} & \\
\hline Faecal characteristics & & & & & & & \\
\hline $\mathrm{pH}$ & 6.66 & 0.52 & 6.96 & 0.68 & 6.94 & 0.65 & 0.200 \\
\hline Moisture (\% of wet weight) & $70 \cdot 65$ & $6 \cdot 90$ & 71.65 & $6 \cdot 25$ & $74 \cdot 10$ & $6 \cdot 47$ & 0.131 \\
\hline
\end{tabular}

a,b Mean or median values within a row with unlike superscript letters were significantly different $(P<0.05)$.

* Sex-, age-, and BMl-adjusted $P$ values.

† Sum of iso-butyrate, iso-valerate and iso-caproic acid.

‡ Sum of valerate, caproic acid and heptanoic acid. 
Table 4. Culture-dependent analysis of gut microbiota (bacteria, yeasts, fungi) ${ }^{*}$

(Mean values and standard deviations; medians and quartiles 1-3 (Q1-Q3); number of positive samples)

\begin{tabular}{|c|c|c|c|c|c|c|c|}
\hline & \multicolumn{6}{|c|}{ Tertiles of MedDietScore } & \multirow[b]{3}{*}{$P_{\text {for trend }} t$} \\
\hline & \multicolumn{2}{|c|}{ Low (n 29) } & \multicolumn{2}{|c|}{ Medium ( $n$ 27) } & \multicolumn{2}{|c|}{ High ( $n$ 36) } & \\
\hline & Mean & SD & Mean & SD & Mean & SD & \\
\hline Total aerobes & $8 \cdot 12$ & 1.07 & $8 \cdot 14$ & 0.88 & 8.02 & 0.79 & $0.888 \dagger$ \\
\hline Total coliforms & $7 \cdot 25$ & 1.19 & 6.98 & 0.90 & 6.83 & 1.05 & $0.287 \dagger$ \\
\hline Escherichia coli & $7 \cdot 24^{a}$ & 1.20 & $6 \cdot 69^{a, b}$ & 1.04 & $6 \cdot 46^{\mathrm{b}}$ & 1.38 & $0.065 \dagger$ \\
\hline Enterococci & 6.08 & $1 \cdot 11$ & $5 \cdot 78$ & 1.73 & 5.96 & 1.31 & $0.727 \dagger$ \\
\hline $\begin{array}{l}\text { Staphylococci } \\
\text { Detection of staphylococci }\end{array}$ & \multicolumn{2}{|c|}{$24^{a, b}$} & \multicolumn{2}{|r|}{1.17} & \multicolumn{2}{|l|}{3.03} & $\begin{array}{l}0.295 \dagger \\
0.028\end{array}$ \\
\hline $\begin{array}{l}\text { Staphylococcus aureus } \\
\text { Detection of } S \text {. aureus }\end{array}$ & \multicolumn{2}{|c|}{$18^{\mathrm{a}, \mathrm{b}}$} & \multicolumn{2}{|c|}{$12^{\mathrm{a}}$} & \multicolumn{2}{|c|}{$26^{\mathrm{b}}$} & $\begin{array}{l}0.175 \dagger \\
0.114\end{array}$ \\
\hline Total anaerobes & $9 \cdot 81^{\mathrm{a}}$ & 0.44 & $9.47^{\mathrm{b}}$ & 0.54 & $9.59^{\mathrm{a}, \mathrm{b}}$ & 0.62 & $0.056 \dagger$ \\
\hline Clostridia & 4.88 & 1.25 & 4.69 & 1.39 & 4.87 & 1.05 & $0.761 \dagger$ \\
\hline Clostridium perfringens & 3.82 & 1.27 & 3.91 & 1.50 & 4.03 & 1.35 & $0.762 \dagger$ \\
\hline Lactobacillus spp. & $5 \cdot 79$ & 1.34 & 5.47 & 1.52 & 5.73 & 1.27 & $0.539 \dagger$ \\
\hline Bifidobacterium spp. & \multirow{2}{*}{\multicolumn{2}{|c|}{9.07}} & & & & & $0.811 \dagger$ \\
\hline Median & & & \multicolumn{2}{|c|}{8.77} & \multicolumn{2}{|c|}{8.78} & \\
\hline Q1-Q3 & \multicolumn{2}{|c|}{$7.39-9.35$} & \multicolumn{2}{|c|}{$7.40-9.38$} & \multicolumn{2}{|c|}{$8 \cdot 34-9.33$} & \\
\hline Bacteroides spp. & 8.37 & 1.23 & 8.46 & 1.04 & 8.43 & 1.26 & $0.933 \dagger$ \\
\hline Yeasts & $3 \cdot 17^{\mathrm{a}, \mathrm{b}}$ & 1.24 & $2 \cdot 69^{\mathrm{a}}$ & 0.84 & $3.56^{\mathrm{b}}$ & 0.92 & $0.016 \dagger$ \\
\hline Detection of yeasts & \multicolumn{2}{|c|}{22} & \multicolumn{2}{|c|}{24} & \multicolumn{2}{|c|}{30} & 0.433 \\
\hline Candida spp.‡ & \multirow{2}{*}{\multicolumn{2}{|c|}{$3 \cdot 15^{\mathrm{a}, \mathrm{b}}$}} & \multicolumn{2}{|l|}{$2 \cdot 62^{\mathrm{a}}$} & \multicolumn{2}{|l|}{$3.39^{\mathrm{b}}$} & $0.055 \dagger$ \\
\hline Detection of Candida spp. & & & \multicolumn{2}{|c|}{19} & \multicolumn{2}{|c|}{29} & 0.444 \\
\hline Candida albicans & $2 \cdot 69^{a}$ & 0.48 & $2 \cdot 99^{a, b}$ & 0.82 & $3 \cdot 28^{b}$ & 0.54 & $0.074 \dagger$ \\
\hline Detection of $C$. albicans & & & & & & & 0.131 \\
\hline Candida glabrata & $2 \cdot 90$ & 1.31 & $2 \cdot 14$ & 0.85 & $2 \cdot 89$ & 0.75 & $0.653 \dagger$ \\
\hline Detection of C. glabrata & & & & & & & 0.972 \\
\hline Candida parapsilosis & $2 \cdot 65$ & 1.31 & 2.04 & 0.56 & 3.14 & 0.96 & $0.664 \dagger$ \\
\hline Detection of C. parapsilosis & & & & & & & 0.795 \\
\hline Candida krusei & 2.96 & 1.01 & $2 \cdot 35$ & 0.81 & 3.06 & 1.19 & $0.463 \dagger$ \\
\hline Detection of $C$. krusei & & & & & & & 0.487 \\
\hline Rhodotorula-like yeasts & 2.72 & 1.01 & $2 \cdot 18$ & 0.42 & 2.55 & 0.73 & $0.309 \dagger$ \\
\hline Detection of Rhodotorula & & & & & & & 0.283 \\
\hline Fungi & & & & & & & $0.978 \dagger$ \\
\hline Median & & & & & & & \\
\hline Q1-Q3 & & & & & & & \\
\hline Detection of fungi & & & & & & & 0.787 \\
\hline Bifidobacteria:E. coli ratio & & & & & & & $0.077 \dagger$ \\
\hline Median & $1 \cdot 16^{\mathrm{a}}$ & & $1 \cdot 30^{\mathrm{a}, \mathrm{b}}$ & & $1 \cdot 24^{\mathrm{b}}$ & & \\
\hline Q1-Q3 & $1.00-1.34$ & & $1.08-1.35$ & & $1.15-1.49$ & & \\
\hline Bacteria:yeasts ratio & $3 \cdot 47^{\mathrm{a}, \mathrm{b}}$ & 0.99 & $3 \cdot 87^{\mathrm{a}}$ & 1.17 & $2 \cdot 91^{\mathrm{b}}$ & 0.78 & $0.006 \dagger$ \\
\hline Bacteria:Candida ratio & $3 \cdot 29^{a, b}$ & 0.96 & $3.89^{\mathrm{a}}$ & 1.22 & $3.05^{\mathrm{b}}$ & 1.02 & $0.032 \dagger$ \\
\hline Bacteria:fungi ratio & 4.40 & 1.45 & 4.40 & 1.14 & $4 \cdot 28$ & 1.49 & $0.995 \dagger$ \\
\hline
\end{tabular}

a,b Mean or median values within a row with unlike superscript letters were significantly different $(P<0.05)$.

* Values are $\log _{10}$ colony-forming units/g wet faeces (counts).

$\dagger$ Sex-, age- and BMI-adjusted $P$ values.

‡ For Candida spp. and species: $n 100$ (low tertile: $n$ 31, medium tertile: $n$ 29, high tertile: $n$ 40).

BMI (Table 6). In specific, MedDietScore was associated positively with faecal moisture, total bacteria levels, bifidobacteria:E. coli ratio (culture based) and the relative share of Bacteroides spp., and negatively with counts of $E$. coli and the molar ratio of valerate after age, sex and BMI adjustment (Table 6). A correlation of MedDietScore with $C$. albicans counts (0.066 (SD 0.033), $P=0.054$ ) and total SCFA concentration (1.554 (SD 0.789), $P=0.052$ ) was also observed after age, sex and BMI adjustment. In terms of gastrointestinal symptomatology, MedDietScore was positively correlated with bloating, sum of symptoms (Table 6) and score of pain (0.173 (SD 0.088), $P=0.054$ ) after age, sex and BMI adjustment.

Though not components of the MedDietScore, consumption of two food groups (snacks and junk food, stimulants) correlated negatively with adherence to the Mediterranean diet in this study and may have significant effects in gut microbiota characteristics. Consumption of snacks and junk food correlated negatively with faecal moisture ( -7.541 (SD 2.569), $P=0.004$ ), Firmicutes ( -0.195 (sD 0.077), $P=0.013$ ), the $C$. coccoides group ( -0.225 (sD 0.092), $P=0.016$ ), the Clostridium leptum group (-0.306 (sD 0.112), $P=0.007), F$. prausnitzii (-0.501 (sD 0.144), $P=0.001$ ), the Lactobacillus group (qPCR; -0.759 (sD 0.333), $P=0.025)$, Bacteroides (qPCR; -0.246 (SD 0.113), $P=0.032$ ) and the bifidobacteria:E. coli ratio (culture based; $-0 \cdot 395$ (sD 0.175), $P=0.027$ ), and positively with the relative share of coliforms (5.903 (sD 2.524), $P=0.022$ ), counts of E. coli (culture based; 1.360 (sD 0.551$), P=0.016$ ), propionate (5.030 (sD 1.877), $P=0.009)$ and iso-valerate (1.338 (sD 0.508), $P=0.010)$ after 
Table 5. Culture-independent analysis of gut microbiota (quantitative PCR) ${ }^{\star}$

(Mean values and standard deviations; medians and quartiles 1-3 (Q1-Q3); percentage of positive samples)

\begin{tabular}{|c|c|c|c|c|c|c|c|}
\hline & \multicolumn{6}{|c|}{ Tertiles of MedDietScore } & \multirow[b]{3}{*}{$P_{\text {for trend }}$} \\
\hline & \multicolumn{2}{|c|}{ Low $(n 31)$} & \multicolumn{2}{|c|}{ Medium ( $n$ 29) } & \multicolumn{2}{|c|}{ High ( $n$ 40) } & \\
\hline & Mean & SD & Mean & SD & Mean & SD & \\
\hline Total bacteria & 11.68 & 0.23 & 11.75 & 0.19 & 11.75 & 0.23 & $0.421 \dagger$ \\
\hline Firmicutes & 11.67 & 0.20 & $11 \cdot 77$ & 0.14 & 11.69 & 0.22 & $0.187 \dagger$ \\
\hline Bacteroidetes & 11.09 & 0.27 & 11.07 & 0.19 & 11.09 & 0.29 & $0.832 \dagger$ \\
\hline Firmicutes:Bacteroidetes ratio & 1.05 & 0.02 & 1.06 & 0.02 & 1.05 & 0.02 & $0.121 \dagger$ \\
\hline Bacteroides spp. & 11.00 & 0.32 & $10 \cdot 98$ & 0.22 & 11.03 & 0.31 & $0.596 \dagger$ \\
\hline Prevotella spp. & & & & & & & $0.623 \dagger$ \\
\hline Median & \multicolumn{2}{|c|}{$9 \cdot 30$} & \multicolumn{2}{|c|}{$9 \cdot 01$} & \multicolumn{2}{|c|}{$8 \cdot 83$} & \\
\hline Q1-Q3 & \multicolumn{2}{|c|}{$8 \cdot 72-10 \cdot 71$} & \multicolumn{2}{|c|}{$8 \cdot 77-10 \cdot 38$} & \multicolumn{2}{|c|}{$8 \cdot 57-10.58$} & \\
\hline$\%$ detection of Prevotella & \multirow{2}{*}{\multicolumn{2}{|c|}{$90 \cdot 3$}} & \multicolumn{2}{|c|}{$79 \cdot 3$} & \multirow{2}{*}{\multicolumn{2}{|c|}{$92 \cdot 5$}} & 0.223 \\
\hline Prevotella:Bacteroides ratio & & & & & & & $0.547 \dagger$ \\
\hline Median & \multicolumn{2}{|c|}{0.89} & \multicolumn{2}{|c|}{0.82} & \multicolumn{2}{|c|}{0.80} & \\
\hline Q1-Q3 & \multicolumn{2}{|c|}{$0.80-0.98$} & \multicolumn{2}{|c|}{$0.79-0.98$} & \multicolumn{2}{|c|}{$0.78-0.97$} & \\
\hline Clostridium coccoides group & $10 \cdot 80$ & 0.26 & $10 \cdot 82$ & 0.22 & $10 \cdot 77$ & 0.24 & $0.829+$ \\
\hline Roseburia spp.-Eubacterium rectale & & & & & & & $0.946 \dagger$ \\
\hline Median & \multicolumn{2}{|c|}{$10 \cdot 42$} & \multicolumn{2}{|c|}{$10 \cdot 38$} & \multicolumn{2}{|c|}{$10 \cdot 31$} & \\
\hline Q1-Q3 & \multicolumn{2}{|c|}{$10 \cdot 10-10 \cdot 53$} & \multicolumn{2}{|c|}{$10 \cdot 19-10 \cdot 54$} & 10 & & \\
\hline $\begin{array}{l}\text { Clostridial cluster IV } \\
\text { (Clostridium leptum group) }\end{array}$ & $10 \cdot 70$ & 0.29 & $10 \cdot 81$ & 0.22 & $10 \cdot 74$ & 0.32 & $0.345 \dagger$ \\
\hline Faecalibacterium prausnitzii & & & & & & & $0.745 \dagger$ \\
\hline Median & & & & & & & \\
\hline Q1-Q3 & 10 & & 10 & & 10 & & \\
\hline C. coccoides: $C$. leptum ratio & 1.01 & 0.03 & 1.00 & 0.02 & 1.00 & 0.02 & $0.292 \dagger$ \\
\hline Clostridium perfringens group & 8.09 & 0.69 & $8 \cdot 11$ & 0.71 & $8 \cdot 20$ & 0.73 & $0.733 \dagger$ \\
\hline Lactobacillus group & 8.05 & 0.70 & $7 \cdot 83$ & 0.92 & $7 \cdot 98$ & 0.85 & $0.549 \dagger$ \\
\hline Bifidobacterium spp. & & & & & & & $0.481 \dagger$ \\
\hline Median & & & & & & & \\
\hline Q1-Q3 & 10 & & & & 10 & & \\
\hline Escherichia coli subgroup & 8.08 & 1.41 & $7 \cdot 79$ & $1 \cdot 14$ & 7.90 & 1.04 & $0.623+$ \\
\hline Bifidobacteria:E. coli ratio & 1.33 & 0.25 & 1.34 & 0.32 & $1 \cdot 34$ & 0.20 & $0.958 \dagger$ \\
\hline Staphylococcus aureus & $4 \cdot 81$ & 0.49 & $4 \cdot 72$ & 0.87 & 4.95 & 0.54 & $0.534 \dagger$ \\
\hline$\%$ detection of $S$. aureus & & & & & & & 0.173 \\
\hline Akkermansia muciniphila & & & & & & & $0.607 \dagger$ \\
\hline Median & & & & & & & \\
\hline Q1-Q3 & & & & & & & \\
\hline$\%$ detection of $A$. muciniphila & & & & & & & 0.440 \\
\hline Methanobrevibacter & & & & & & & $0.549 \dagger$ \\
\hline Median & & & & & & & \\
\hline Q1-Q3 & & & & & & & \\
\hline$\%$ detection of methanogens & & & & & & & 0.635 \\
\hline
\end{tabular}

* Values are $\log _{10}$ copies of $16 \mathrm{~S}$ rRNA gene/g wet faeces (levels) or $\log _{10}$ copies of nuc gene/g wet faeces for $S$. aureus.

† Sex-, age- and BMl-adjusted $P$ values.

Table 6. Results from simple correlation analysis and multiple linear regression models for adherence to Mediterranean diet (MedDietScore) and gastrointestinal, faecal and gut microbiota characteristics of the participants

$(\beta$-Coefficients with their standard errors)

\begin{tabular}{|c|c|c|c|c|c|}
\hline \multirow[b]{2}{*}{ Dependent outcomes } & \multirow[b]{2}{*}{ Independent factor } & \multirow{2}{*}{$\frac{\text { Correlation coefficient }}{(r \text { and } P \text { value })}$} & \multicolumn{3}{|c|}{ Sex-, age- and BMI-adjusted models } \\
\hline & & & $\beta$-Coefficient & SE & $P$ \\
\hline$\%$ faecal moisture & MedDietScore (per 1 point) & $r 0.235, P=0.019$ & 0.357 & $0 \cdot 167$ & 0.035 \\
\hline Total bacteria (qPCR) & MedDietScore (per 1 point) & $r 0.250, P=0.012$ & 0.014 & 0.006 & 0.016 \\
\hline Escherichia coli (culture based) & MedDietScore (per 1 point) & $r-0.317, P=0.003$ & -0.111 & 0.037 & 0.004 \\
\hline Bifidobacteria:E. coli (culture based) & MedDietScore (per 1 point) & $r 0.313, P=0.004$ & 0.035 & 0.012 & 0.003 \\
\hline Relative share of bacteroides & MedDietScore (per 1 point) & $r 0.210, P=0.011$ & 1.310 & 0.473 & 0.007 \\
\hline Molar ratio of valerate & MedDietScore (per 1 point) & $r-0.195, P=0.052$ & -0.042 & 0.021 & 0.049 \\
\hline Bloating & MedDietScore (per 1 point) & $r 0.311, P=0.002$ & 0.197 & 0.095 & 0.042 \\
\hline Sum of symptoms & MedDietScore (per 1 point) & $r 0.258, P=0.011$ & 0.766 & 0.315 & 0.017 \\
\hline
\end{tabular}

qPCR, quantitative PCR.

sex, age and BMI adjustment. Stimulant consumption was negatively associated with stool $\mathrm{pH}(-0.360$ (SD 0.143$)$, $P=0.013$ ), $S$. aureus (qPCR; -0.573 (sD 0.251), $P=0.026$ ) and staphylococci counts (culture based; -0.517 (SD 0.239), $P=0.034$ ) after sex, age and BMI adjustment. Further analysis into the stimulant food group revealed that these effects were 
attributable exclusively to coffee or tea consumption and that the consumption of sodas was correlated negatively with faecal levels of $A$. muciniphila ( -3.717 (sD 1.641), $P=0.026$ ).

\section{Discussion}

The present study aimed to explore possible associations of adherence to the Mediterranean diet with the gut microbiota profile and gastrointestinal symptoms in an adult population. Regarding the well-documented geographical and ethnic variation of gut microbial composition in humans ${ }^{(34)}$, similar scientific efforts may contribute to validation of the robustness of the proposed microbial indicators of metabolic health and inflammation. Moreover, the influence of the Mediterranean diet in gut microbial ecology is currently revealed ${ }^{(12-15,18,19)}$, and this scientific field is open to further investigation. Thus, our findings indicate that a high adherence to the Mediterranean diet was characterised by lower $E$. coli counts and a subsequently higher culture-based bifidobacteria:E. coli ratio, increased levels and prevalence of $C$. albicans, greater molar ratio of acetate, higher defaecation frequency and a more pronounced gastrointestinal symptomatology compared with the low tertile. An overall lower molar ratio of valerate in the case of high adherence to the Mediterranean diet compared with other score levels was also indicated. Positive correlations of MedDietScore with gastrointestinal symptoms, faecal moisture, total bacteria, bifidobacteria:E. coli ratio, relative share of bacteroides, C. albicans and total SCFA, and negative associations with cultivable $E$. coli levels and valerate were also indicated. Further associations between gut microbiota characteristics and consumption of snacks and junk food or stimulants were also revealed.

Beneficial effects of the Mediterranean diet in the management of chronic diseases are attributed to the cumulative synergistic and interactive combinations of nutrients ${ }^{(10)}$. Characteristics of the Mediterranean diet, such as intake of non-refined cereals, vegetables, fruit, olive oil and red wine, are linked to a great repertoire of constituents with potential effects in gut microbiota dynamics $^{(17)}$. In the present study, positive associations of MedDietScore with total bacteria and bacteroides characteristics could be attributed to the high carbohydrate, fibre, unsaturated lipid and antioxidant content of the Mediterranean diet, as previously reported ${ }^{(13-14,17)}$. High adherence to the Mediterranean diet was also related to decreased counts of $E$. coli, a representative pathogenic bacterium, and subsequently to an increased ratio of typical beneficial bifidobacteria: $E$. coli, which is considered an important indicator for gut microbiota equilibrium and overall health ${ }^{(35)}$. Long-term polysaccharide-rich diets have been linked to underrepresentation of Enterobacteriaceae (Shigella and Escherichia), and dietary antioxidants may inhibit the growth of $E$. coli strains ${ }^{(18,36-38)}$.

Previously reported connections of the Mediterranean $\operatorname{diet}^{(12-15,19)}$ or plant-based diets typical in rural, agrarian societies $^{(39)}$ with suggested diet-responsive gut microbiota characteristics (e.g. Prevotella, Prevotella:Bacteroides ratio, Roseburia-E. rectale) were not found in the current work. Roager et al. ${ }^{(40)}$ also reported stable Prevotella:Bacteroides ratio in Danish subjects with central obesity and components of the metabolic syndrome after a controlled intervention with the
New Nordic Diet, which includes more fruits, vegetables and whole grains, and less added sugar and saturated fat. Factors related to the urban setting of the present research, the variation in fibre, protein and fat content as well as the quality of diets among studies, the differences in definition of adherence level to the Mediterranean diet or even the reported underrepresentation of the Prevotella group in Nordic and Southern European countries could be the reason for these discrepancies $^{(12,39,41)}$.

The diversity of the human gut mycobiome remains poorly explored $^{(42)}$ and available data about the Mediterranean diet and gut eukaryotes are rather scarce. In this study, high adherence to the Mediterranean diet was characterised by increased $C$. albicans colonisation patterns. Candida and other yeasts are associated with features of $\operatorname{diet}^{(43,44)}$, and the connection of high adherence with increased levels of yeasts could be attributed to ingestion of foodstuff that are carriers of yeasts, such as fruits, juices and fermented food products ${ }^{(44,45)}$.

SCFA are major gut microbial metabolites with a high potential impact on host molecular mechanisms because of their role as substrates and/or signalling molecules ${ }^{(46)}$. Increased amount of faecal SCFA was also previously observed in high-level adherence to the Mediterranean diet ${ }^{(12-13,19)}$, a fact that could be interpreted by the enhanced microbial-dependent fermentation of indigestible carbohydrates reaching the colon $^{(46)}$. Host factors such as transit time may also have a pivotal role in the total amount of SCFA excreted in faeces ${ }^{(47)}$, which could merely justify suppressed SCFA levels observed in the medium tertile. Connections of adherence to the Mediterranean diet with acetate and other SCFA levels (e.g. valerate) were also previously reported ${ }^{(12)}$ and reflected differences in consumption of plant- and animal-origin food groups, whereas positive relations with propionate and butyrate ${ }^{(12-13)}$ were not replicated in this study, possibly because of the influence of known factors that affect the human gut-associated metabolome, such as sex and age ${ }^{(12)}$.

A high adherence to the Mediterranean diet was related to higher stool frequency and faecal moisture and characterised by greater, though mild, gastrointestinal symptomatology. Dietary fibre consumption, fermentation and bulking-effect caused directly via water retention are significant contributors in these relationships ${ }^{(48)}$. Furthermore, the lipid content of olive oil, a core constituent of the Mediterranean diet, could exert a lubricant and stool-softening effect and enhance important stimuli for bowel movements through interactions with bile acids ${ }^{(49)}$.

In the present study, intriguing associations between gut microbiota characteristics and consumption of stimulants or snacks and junk food were found. The inverse relation of stimulant consumption with the prevalence of faecal $S$. aureus could be attributed to more acidic stool $\mathrm{pH}$ and to the potential systemic antimicrobial activity of coffee and tea polyphenols against a wide range of pathogenic micro-organisms ${ }^{(50)}$. The negative association of soda consumption with levels of A. muciniphila, a mucin-degrading bacterium with possible beneficial effects against obesity and type 2 diabetes ${ }^{(4,51,52)}$, raises further interest. Higher consumption of snack and junk food products was characterised by increased counts of E. coli and suppressed presence of lactobacilli and butyrate-producing 
Firmicutes members (e.g. C. coccoides group, C. leptum group, $F$. prausnitzii), resulting in a potential detrimental inflammatory gut microbiota milieu for the host ${ }^{(1,5,53)}$. Furthermore, increased faecal levels of the branched SCFA iso-valerate may reflect bacterial catabolism of animal protein ${ }^{(54)}$, whereas elevated amount of propionate in faeces may result from increased dietary intake of propionate salts, common preservatives in the food industry, and may exert versatile effects on host physiology and pathology ${ }^{(55)}$. Though evidence regarding junk food consumption and gut microbiota profiling in humans is very limited, in animal models it has been documented that following a Westernised 'fast food' style diet or a Western-style high-energy cafeteria diet results in restructuring of the gut microbiota ${ }^{56,57)}$. Moreover, anecdotal experimental data have proposed the devastating effects of an exclusively fast food diet on the human gut microbiome diversity, with a $40 \%$ reduction in detectable species within $10 \mathrm{~d}$ of consumption ${ }^{(58,59)}$. Finally, cumulative evidence suggests that following a Western-type, high-fat, refined-carbohydrate-rich diet and frequent consumption of highly processed and preserved foods, which reduce the intake of commensal, food-associated microbes, could disturb the gut microbiota balance and deserves special attention ${ }^{(60,61)}$.

In this study, a detailed record of dietary, exercise, lifestyle and gastrointestinal parameters allowed the in-depth assessment of the population under investigation, whereas analysis adjustments for factors with established effects on gut microbiota composition (e.g. sex, age, BMI) allowed to explore the contribution of possible covariates ${ }^{(6,34)}$. The exclusion of LER from the analyses could minimise the systematic error of under-reporting. Even though microbiota sequencing was not available in this study, qPCR methodology in combination with cultivation techniques provided a thorough analysis of the gut microbiome and mycobiome. On the other hand, the cross-sectional study design undermines the causality of the reported results and future prospective and intervention studies are essential.

The use of MedDietScore for the assessment of adherence to the Mediterranean diet may also have some limitations. No weighting has been applied to the components of the score, mainly because it is hard to select the best weight because of the lack of sufficient data to support components' weighting (i.e. from meta-analyses on the specific components). Thus, we cannot exclude the possibility that two individuals may have the same score but different dietary intakes. This is an inherent limitation of the composite diet scores presented in the literature. Moreover, we could not use a priori-defined cut-off points for the adherence (or non-adherence) to the Mediterranean diet, because the Mediterranean diet as a healthy prototype should be followed in total. The cut-off points used here were the tertiles of the MedDietScore; by dividing the group into three equal-sized subgroups, optimal statistical power is achieved. This approach has been routinely used in observational studies investigating the role of adherence to the Mediterranean diet in health status ${ }^{(22,23,62-64)}$. It is true that the cut-off points used in MedDietScore for the description of the characteristics in our sample were data driven. This means that the 'low' cut-off point of the MedDietScore could be different in another population group. However, the classification of MedDietScore was used only for descriptive results, whereas continuous values of the score were used in all multi-adjusted statistical analyses. Thus, the findings of diet-outcome(s) relationships and their significance were not related to the thresholds used for the tertiles of the MedDietScore. Finally, it is interesting to note that in our study, the mean MedDietScore was comparable with values reported in other studies performed outside Greece (e.g. USA, UK), in which adherence to the Mediterranean diet was calculated on the basis of the same diet score as in our research ${ }^{(62-64)}$. In detail, MedDietScore ranged from 18 to 46 in the study by Tangney et al. ${ }^{(64)}$, with a mean value of 26 for the low tertile, 31 for the medium tertile and 37 for the high tertile, whereas in the study by Koyama et $a l^{(62)}$, racespecific tertiles for Whites were 12-29 (low), 30-34 (medium) and 35-50 (high tertile). Thus, our data might be applied to other populations beyond Greek individuals and these observations could add considerable strength to the argument about the 'health' aspect of the Mediterranean diet.

In conclusion, our findings support a link between adherence to the Mediterranean diet and the gut microbiota profile, SCFA production and gastrointestinal symptoms. Additional research is necessary to elucidate connections of the Mediterranean food pattern with gut microbiota characteristics, possibly under the prism of other long-term dietary habits (e.g. stimulant and fast food consumption).

\section{Acknowledgements}

This work was supported by TANITA Healthy Weight Community Trust (2013 and 2015 Grants) and the Master of Science Programme of Applied Nutrition and Dietetics, Harokopio University. TANITA Healthy Weight Community Trust and Master of Science Programme of Applied Nutrition and Dietetics had no role in the design, analysis or writing of this article.

E. K. M. and A. K. contributed to formulating the research questions, designing the study, analysing the data and writing the article; E. K. M. and A. K. contributed to carrying out the study; D. B. P. and K. C. M. contributed to analysing the data and writing the article; M. Y. and S. A. contributed to writing the article.

None of the authors has any conflicts of interest to declare.

\section{Supplementary material}

For supplementary material/s referred to in this article, please visit https://doi.org/10.1017/S0007114517001593

\section{References}

1. Sekirov I, Russell SL, Antunes LC, et al. (2010) Gut microbiota in health and disease. Physiol Rev 90, 859-904.

2. Tremaroli V \& Bäckhed F (2012) Functional interactions between the gut microbiota and host metabolism. Nature 489, 242-249.

3. Bifulco M (2015) Mediterranean diet: the missing link between gut microbiota and inflammatory diseases. Eur J Clin Nutr 69, 1078.

4. Dao MC, Everard A, Aron-Wisnewsky J, et al. (2016) Akkermansia muciniphila and improved metabolic health during a dietary intervention in obesity: relationship with gut microbiome richness and ecology. Gut 65, 426-436.

5. Miquel S, Martín R, Rossi O, et al. (2013) Faecalibacterium prausnitzii and human intestinal health. Curr Opin Microbiol 16, 255-261. 
6. Stenman LK, Burcelin R \& Lahtinen S (2015) Establishing a causal link between gut microbes, body weight gain and glucose metabolism in humans - towards treatment with probiotics. Benef Microbes 13, 1-12.

7. Moschen AR, Wieser V \& Tilg H (2012) Dietary factors: major regulators of the gut's microbiota. Gut Liver 6, 411-416.

8. Sofi F, Cesari F, Abbate R, et al. (2008) Adherence to Mediterranean diet and health status: meta-analysis. BMJ 337, a1344.

9. Willett WC, Sacks F, Trichopoulou A, et al. (1995) Mediterranean diet pyramid: a cultural model for healthy eating. Am J Clin Nutr 61, Suppl. 6, S1402-S1406.

10. Del Chierico F, Vernocchi P, Dallapiccola B, et al. (2014) Mediterranean diet and health: food effects on gut microbiota and disease control. Int J Mol Sci 15, 11678-11699.

11. Schröder H (2007) Protective mechanisms of the Mediterranean diet in obesity and type 2 diabetes. J Nutr Biochem 18, 149-160.

12. De Filippis F, Pellegrini N, Vannini L, et al. (2015) High-level adherence to a Mediterranean diet beneficially impacts the gut microbiota and associated metabolome. Gut (Epublication ahead of print version 28 September 2015).

13. Gutiérrez-Díaz I, Fernández-Navarro T, Sánchez B, et al. (2016) Mediterranean diet and faecal microbiota: a transversal study. Food Funct 7, 2347-2356.

14. Haro C, Garcia-Carpintero S, Alcala-Diaz JF, et al. (2016) The gut microbial community in metabolic syndrome patients is modified by diet. $J$ Nutr Biochem 27, 27-31.

15. Haro C, Montes-Borrego M, Rangel-Zúñiga OA, et al. (2016) Two healthy diets modulate gut microbial community improving insulin sensitivity in a human obese population. J Clin Endocrinol Metab 101, 233-242.

16. Kouris-Blazos A \& Itsiopoulos C (2014) Low all-cause mortality despite high cardiovascular risk in elderly Greek-born Australians: attenuating potential of diet? Asia Pac J Clin Nutr 23, 532-544.

17. Lopez-Legarrea P, Fuller NR, Zulet MA, et al. (2014) The influence of Mediterranean, carbohydrate and high protein diets on gut microbiota composition in the treatment of obesity and associated inflammatory state. Asia Pac J Clin Nutr 3, 360-368.

18. Marlow G, Ellett S, Ferguson IR, et al. (2013) Transcriptomics to study the effect of a Mediterranean-inspired diet on inflammation in Crohn's disease patients. Hum Genomics 7, 24.

19. Shankar V, Gouda M, Moncivaiz J, et al. (2017) Differences in gut metabolites and microbial composition and functions between Egyptian and U.S. children are consistent with their diets. mSystems 2, e00169-16.

20. Fountoulakis KN, Bech P, Panagiotidis P, et al. (2007) Comparison of depressive indices: reliability, validity, relationship to anxiety and personality and the role of age and life events. J Affect Disord 97, 187-195.

21. Bountziouka V, Bathrellou E, Giotopoulou A, et al. (2012) Development, repeatability and validity regarding energy and macronutrient intake of a semi-quantitative food frequency questionnaire: methodological considerations. Nutr Metab Cardiovasc Dis 22, 659-667.

22. Panagiotakos DB, Pitsavos C \& Stefanadis C (2006) Dietary patterns: a Mediterranean diet score and its relation to clinical and biological markers of cardiovascular disease risk. Nutr Metab Cardiovasc Dis 16, 559-568.

23. Pitsavos C, Panagiotakos DB, Tzima N, et al. (2005) Adherence to the Mediterranean diet is associated with total antioxidant capacity in healthy adults: the ATTICA study. $A m \mathrm{~J}$ Clin Nutr 82, 694-699.

24. FAO/WHO/UNU (2005) Human energy requirements: report of a joint FAO/WHO/UNU expert consultation. Food Nutr Bull 26, 166.
25. Goldberg GR, Black AE, Jebb SA, et al. (1991) Critical evaluation of energy intake data using fundamental principles of energy physiology: 1 . Derivation of cut-off limits to identify under-recording. Eur J Clin Nutr 45, 569-581.

26. Papathanasiou G, Georgoudis G, Papandreou M, et al. (2009) Reliability measures of the short International Physical Activity Questionnaire (IPAQ) in Greek young adults. Hellenic J Cardiol 50, 283-294.

27. Kajander K, Hatakka K, Poussa T, et al. (2005) A probiotic mixture alleviates symptoms in irritable bowel syndrome patients: a controlled 6-month intervention. Aliment Pharmacol Ther 22, 387-394.

28. Lewis SJ \& Heaton KW (1997) Stool form scale as a useful guide to intestinal transit time. Scand J Gastroenterol 32, 920-924.

29. Bovenschen HJ, Janssen MJR, van Oijen MGH, et al. (2006) Evaluation of a gastrointestinal symptoms questionnaire. Dig Dis Sci 51, 1509-1515.

30. Sepp E, Lõivukene K, Julge K, et al. (2013) The association of gut microbiota with body weight and body mass index in preschool children of Estonia. Microbial Ecol Health Dis 24, 19231.

31. Salonen A, Nikkilä J, Jalanka-Tuovinen J, et al. (2010) Comparative analysis of fecal DNA extraction methods with phylogenetic microarray: effective recovery of bacterial and archaeal DNA using mechanical cell lysis. J Microbiol Methods 81, $127-134$.

32. Mitsou EK, Kougia E, Nomikos T, et al. (2011) Effect of banana consumption on faecal microbiota: a randomised, controlled trial. Anaerobe 17, 384-387.

33. Turunen K, Tsouvelakidou E, Nomikos T, et al. (2011) Impact of beta-glucan on the faecal microbiota of polypectomized patients: a pilot study. Anaerobe 17, 403-406.

34. Mueller S, Saunier K, Hanisch C, et al. (2006) Differences in fecal microbiota in different European study populations in relation to age, gender, and country: a cross-sectional study. Appl Environ Microbiol 72, 1027-1033.

35. Gao X, Jia R, Xie L, et al. (2015) Obesity in school-aged children and its correlation with gut E.coli and Bifidobacteria: a case-control study. BMC Pediatr 15, 64

36. De Filippo C, Cavalieri D, Di Paola M, et al. (2010) Impact of diet in shaping gut microbiota revealed by a comparative study in children from Europe and rural Africa. Proc Natl Acad Sci U S A 107, 14691-14696.

37. Duda-Chodak A, Tarko T, Satora P, et al. (2015) Interaction of dietary compounds, especially polyphenols, with the intestinal microbiota: a review. Eur J Nutr 54, 325-341.

38. Ferrocino I, Di Cagno R, De Angelis M, et al. (2016) Fecal microbiota in healthy subjects following omnivore, vegetarian and vegan diets: culturable populations and rRNA DGGE profiling. PLOS ONE 10, e0128669.

39. Gorvitovskaia A, Holmes SP \& Huse SM (2016) Interpreting Prevotella and Bacteroides as biomarkers of diet and lifestyle. Microbiome 4, 15.

40. Roager HM, Licht TR, Poulsen SK, et al. (2014) Microbial enterotypes, inferred by the prevotella-to-bacteroides ratio, remained stable during a 6-month randomized controlled diet intervention with the new Nordic diet. Appl Environ Microbiol 80, 1142-1149.

41. Lahti L, Salojärvi J, Salonen A, et al. (2014) Tipping elements in the human intestinal ecosystem. Nat Commun 5, 4344.

42. Mar Rodríguez M, Pérez D, Javier Chaves F, et al. (2015) Obesity changes the human gut mycobiome. Sci Rep 5, 14600.

43. Hoffmann C, Dollive S, Grunberg S, et al. (2013) Archaea and fungi of the human gut microbiome: correlations with diet and bacterial residents. PLOS ONE 8, e66019. 
44. Wirth F \& Goldani LZ (2012) Epidemiology of Rhodotorula: an emerging pathogen. Interdiscip Perspect Infect Dis 2012, 465717.

45. Moreira SR, Schwan RF, de Carvalho EP, et al. (2001) Isolation and identification of yeasts and filamentous fungi from yogurts in Brazil. Braz J Microbiol 32, 117-122.

46. Neves AL, Chilloux J, Sarafian MH, et al. (2015) The microbiome and its pharmacological targets: therapeutic avenues in cardiometabolic diseases. Curr Opin Pharmacol 25, 36-44.

47. Oufir LE, Barry JL, Flourié B, et al. (2000) Relationships between transit time in man and in vitro fermentation of dietary fiber by fecal bacteria. Eur J Clin Nutr 54, 603-609.

48. Eswaran S, Muir J \& Chey WD (2013) Fiber and functional gastrointestinal disorders. Am J Gastroenterol 108, 718-727.

49. Ramos CI, Andrade de Lima AF, Grilli DG, et al. (2015) The short-term effects of olive oil and flaxseed oil for the treatment of constipation in hemodialysis patients. J Ren Nutr 25, 50-56.

50. Matheson EM, Mainous AG 3rd, Everett CJ, et al. (2011) Tea and coffee consumption and MRSA nasal carriage. Ann Fam Med 9, 299-304.

51. Everard A, Belzer C, Geurts L, et al. (2013) Cross-talk between Akkermansia muciniphila and intestinal epithelium controls diet-induced obesity. Proc Natl Acad Sci USA 110, 9066-9071.

52. Karlsson CL, Onnerfält J, Xu J, et al. (2012) The microbiota of the gut in preschool children with normal and excessive body weight. Obesity 20, 2257-2261.

53. Collado MC, Isolauri E, Laitinen K, et al. (2010) Effect of mother's weight on infant's microbiota acquisition, composition, and activity during early infancy: a prospective follow-up study initiated in early pregnancy. Am J Clin Nutr 92, 1023-1030.

54. Ríos-Covián D, Ruas-Madiedo P, Margolles A, et al. (2016) Intestinal short chain fatty acids and their link with diet and human health. Front Microbiol 7, 185.
55. Al-Lahham SH, Peppelenbosch MP, Roelofsen H, et al. (2010) Biological effects of propionic acid in humans; metabolism, potential applications and underlying mechanisms. Biochim Biophys Acta 1801, 1175-1183.

56. Kaakoush NO, Martire SI, Raipuria M, et al. (2017) Alternating or continuous exposure to cafeteria diet leads to similar shifts in gut microbiota compared to chow diet. Mol Nutr Food Res 61, 1500815

57. Poutahidis T, Kleinewietfeld M, Smillie C, et al. (2013) Microbial reprogramming inhibits Western diet-associated obesity. PLOS ONE 8, e68596.

58. Sargent J (2016) In focus: diets, genes, and microbes. Lancet 4, 307.

59. Spector T (2015) The Diet Myth: The Real Science Behind What We Eat. London: Weidenfeld and Nicolson Publishing.

60. Chassaing B, Koren O, Goodrich JK, et al. (2015) Dietary emulsifiers impact the mouse gut microbiota promoting colitis and metabolic syndrome. Nature 519, 92-96.

61. Graf D, Di Cagno R, Fåk F, et al. (2015) Contribution of diet to the composition of the human gut microbiota. Microb Ecol Health Dis 26, 26164.

62. Koyama A, Houston DK, Simonsick EM, et al. (2015) Association between the Mediterranean diet and cognitive decline in a biracial population. J Gerontol A Biol Sci Med Sci 70, 354-359.

63. Papadaki A, Wood L, Sebire SJ, et al. (2015) Adherence to the Mediterranean diet among employees in South West England: formative research to inform a web-based, work-place nutrition intervention. Prev Med Rep 2, 223-228.

64. Tangney CC, Li H, Wang Y, et al. (2014) Relation of DASHand Mediterranean-like dietary patterns to cognitive decline in older persons. Neurology 83, 1410-1416. 